\title{
INTEGRAL long-term monitoring of the supergiant fast X-ray transient XTE J1739-302
}

\author{
P. Blay ${ }^{1}$, S. Martínez-Núñez ${ }^{2,1}$, I. Negueruela ${ }^{2}$, K. Pottschmidt ${ }^{3,4}$, D. M. Smith ${ }^{5}$, J. M. Torrejón ${ }^{2,6}$, P. Reig ${ }^{7,8}$, \\ P. Kretschmar ${ }^{9}$, and I. Kreykenbohm ${ }^{10,11}$ \\ 1 Institut de Ciència dels Materials, Universitat de València, PO Box 22085, 46071 Valencia, Spain \\ e-mail: pere.blay@uv.es \\ 2 Departamento de Física, Ingeniería de Sistemas y Teoría de la Señal, Universidad de Alicante, Apdo. 99, 03080 Alicante, Spain \\ e-mail: ignacio@dfists.ua.es \\ 3 CRESST \& Department of Physics, University of Maryland Baltimore County, 1000 Hilltop Circle, Baltimore, MD 21250, USA \\ 4 NASA Goddard Space Flight Center, Astrophysics Science Division, Code 661, Greenbelt, MD 20771, USA \\ 5 Department of Physics and Santa Cruz Institute for Particle Physics, University of California, Santa Cruz, Santa Cruz, \\ CA 95064, USA \\ ${ }^{6}$ Massachusetts Institute of Technology, Kavli Institute for Astrophysics and Space Research, Cambridge MA 02139, USA \\ 7 IESL, Foundation for Research and Technology, 71110 Heraklion, Crete, Greece \\ 8 Physics Department, University of Crete, PO Box 2208, 71003 Heraklion, Crete, Greece \\ 9 ESA, ESAC, PO Box 78, 28691 Villanueva de la Cañada, Madrid, Spain \\ 10 Institut für Astronomie und Astrophysik, Abteilung Astronomie, Sand 1, 72076 Tübingen, Germany \\ 11 INTEGRAL Science Data Centre, 16 Ch. d'Ecogia, 1290 Versoix, Switzerland
}

Received 10 January 2008 / Accepted 19 June 2008

\section{ABSTRACT}

\begin{abstract}
Context. In the past few years, a new class of high mass X-ray binaries (HMXRB) has been claimed to exist, the supergiant fast X-ray transients (SFXT). These are X-ray binary systems with a compact companion orbiting a supergiant star which show very short and bright outbursts in a series of activity periods overimposed on longer quiescent periods. Only very recently the first attempts to model the behaviour of these sources have been published, some of them within the framework of accretion from clumpy stellar winds.

Aims. Our goal is to analyze the properties of XTE J1739-302/IGR J17391-3021 within the context of the clumpy structure of the supergiant wind.

Methods. We have used INTEGRAL and RXTE/PCA observations to obtain broad band (1-200 keV) spectra and light curves of XTE J1739-302 and investigate its X-ray spectrum and temporal variability.

Results. We have found that XTE J1739-302 follows a much more complex behaviour than expected. Far from presenting a regular variability pattern, XTE J1739-302 shows periods of high, intermediate, and low flaring activity.
\end{abstract}

Key words. binaries: close - supergiants $-\mathrm{X}$-rays: binaries

\section{Introduction}

Wind-fed supergiant X-ray binaries (SGXRBs) display high energy emission arising from the accretion of material in the wind of an OB supergiant onto the compact component of the system (a neutron star - NS - or black hole in orbit around the supergiant). SGXRBs are persistent X-ray sources, displaying an X-ray luminosity $L_{\mathrm{X}} \sim 10^{36} \mathrm{erg} \mathrm{s}^{-1}$. Because of the physical characteristics of wind accretion, their emission is variable on short timescales, with frequent flares, but relatively stable in the long term (for example, the long-term $R X T E / A S M$ lightcurve of Vela X-1, averaged and smoothed with a running window of $30 \mathrm{~d}$ length, shows variations by only a factor of $\sim 4$; Ribó et al. 2006). If the orbit is eccentric, the luminosity is modulated by the orbital period of the system (e.g., Leahy 2002). Stronger short flares, with a fast rise and a typical timescale of the order of a few hours, have been observed from several systems, such as Vela X-1 (Laurent et al. 1995; Krivonos et al. 2003) or 4U 1907+09 (Fritz et al. 2006).

Recently, thanks to the improved sensitivity of high energy missions, many new SGXRBs have been discovered, leading to the suggestion of new classes of X-ray sources. On the one hand, there is a number of highly absorbed SGXRBs, invisible to previous missions due to high absorption in the softer X-ray bands (e.g., Chaty \& Filliatre 2005). On the other hand, supergiant fast X-ray transients (SFXTs) display fast outbursts, with a typical duration of a few hours, but stay in quiescence most of the time (Smith et al. 2006; Negueruela et al. 2006a; Sguera et al. 2006). Unlike in classical SGXRBs, the X-ray luminosity of SFXTs goes below the sensitivity limit of the INTErnational GammaRay Astrophysics Laboratory (INTEGRAL) and they remain undetectable for long time spans. They can only be observed during an outburst or flare, for a short time. Though several models have been proposed for this difference in behaviours, it seems to be a natural consequence of the clumpy nature of $\mathrm{OB}$ star winds (Walter \& Zurita Heras 2007; Negueruela et al. 2008). Sidoli et al. (2007) propose an alternative hypothesis, based on observations of IGR J11215-5952, in which the observed flaring activity is due to the interaction of the compact object with an extended equatorial decretion disc around the supergiant star.

Although the definition of SFXTs as a putative new class of objects was only possible when the optical counterparts to these 
systems started to be identified, INTEGRAL has contributed decisively to the characterization of the high energy behaviour of these sources. So far, $\sim 12$ SFXTs or related objects have been detected by INTEGRAL (Walter \& Zurita Heras 2007; Sguera et al. 2006). Among them, the best characterized system is XTE J1739-302 = IGR J17391-3021, generally taken as the prototype of the class (Smith et al. 2006; Negueruela et al. 2006b).

XTE J1739-302 was discovered by RXTE during a short outburst in 1997 (Smith et al. 1998), when it was detected only for a period of a few hours. The source spectrum was well described by bremsstrahlung emission with a source temperature $k T \sim 21 \mathrm{keV}$. No indications of any periodicity shorter than $300 \mathrm{~s}$ could be found. During 2003, the source was detected by INTEGRAL/ISGRI (Lutovinov et al. 2005). Again, a bremsstrahlung model with $k T \sim 22 \mathrm{keV}$ fitted the source spectrum well and no evidence of periodicity could be found. A total of 6 outbursts were detected by INTEGRAL up to 2005 (Sguera et al. 2005). The mean duration of these outbursts is of the order of 5 hours and they are all highly structured. For a plot of INTEGRAL/ISGRI detections in the $20-40 \mathrm{keV}$ energy range during the period 2003-2005, see Fig. 1. The optical counterpart was identified thanks to a Chandra localization as an O8 Iab(f) supergiant at a distance of $\approx 2.3 \mathrm{kpc}$ (Negueruela et al. 2006b).

In this work, we present a detailed analysis of INTEGRAL data for XTE J1739-302 obtained mostly through the Galactic Plane Scans (GPS, public data), the Galactic Center Deep Exposure (GCDE, public data), and through three long exposures of the Galactic Center, taken as part of the INTEGRAL Key Programme (KP) observations. Section 2 is devoted to the description of the data and the analysis techniques used, including the presentation of results. An interpretation of these results in the context of our current understanding of SFXTs and models of accretion from clumpy winds (Walter \& Zurita Heras 2007; Negueruela et al. 2008) is presented in Sect. 3, followed by our conclusions.

\section{Observations, data reduction and analysis}

INTEGRAL is an ESA mission with contributions from Russia and NASA (Winkler et al. 2003). There are 3 high energy instruments on board INTEGRAL. The SPectrometer on INTEGRAL (SPI, see Vedrenne et al. 2003), the Imager on Board the INTEGRAL Satellite (IBIS, see Ubertini et al. 2003), and the Joint-European X-ray Monitor (JEM-X, Lund et al. 2003). IBIS has two detector layers, ISGRI (INTEGRAL Soft Gamma-Ray Imager, Lebrun et al. 2003) and PICsIT (Pixellated Imaging Caesium Iodide Telescope, Labanti et al. 2003), operating in the $15-1000 \mathrm{keV}$ and $1750 \mathrm{keV}-10 \mathrm{MeV}$ energy ranges respectively. Data from both layers can be combined in the IBIS Compton mode (Lei et al. 1997; Forot et al. 2007). In this work we make use of INTEGRAL/IBIS/ISGRI data (referred to here as INTEGRAL/ISGRI). Data from the INTEGRAL/JEM-X instrument are sparse and only a few detections will be reported.

Exposures including XTE J1739-302 were obtained in September-October 2006 and February-March 2007 (AO-4 Galactic Center Key Programme, from now on KP1, observations) and August-September 2007 (AO-5 Galactic Center Key Programme, from now on KP2, observations). The data analyzed correspond to INTEGRAL revolutions 478-481, 484, 485 (KP1, MJD 53 990-54014), 534-537, 539-542 (KP1, MJD 54 15754 184) and 594-596, 599-601, 604 (KP2, MJD 54 337-54369). A revolution lasts for $\sim 3$ days and observations within a revolution are divided in exposures of the order of $\sim 2 \mathrm{ks}$ (called science
Table 1. Summary of INTEGRAL/JEM-X detections during GCDE and KP1 periods.

\begin{tabular}{lccc}
\hline \hline $\begin{array}{l}\text { INTEGRAL } \\
\text { pointing }\end{array}$ & MJD & $\begin{array}{c}\text { Count Rate } \\
4-15 \mathrm{keV} \\
\left(\text { count s }^{-1}\right)\end{array}$ & $\begin{array}{c}\text { Count Rate } \\
15-30 \mathrm{keV} \\
\left(\text { count s}^{-1}\right)\end{array}$ \\
\hline 00530065 & 52720.54 & $7.1 \pm 1.3$ & $1.7 \pm 0.5$ \\
00530066 & 52720.56 & $8.1 \pm 0.5$ & $1.9 \pm 0.2$ \\
01710057 & 53073.29 & $4.0 \pm 0.4$ & $0.7 \pm 0.3$ \\
01710067 & 53073.53 & $7.7 \pm 1.8$ & $1.9 \pm 0.8$ \\
01710077 & 53073.77 & $2.1 \pm 0.3$ & $3.8 \pm 0.1$ \\
\hline 04850027 & 54011.67 & $3.2 \pm 0.3$ & $0.6 \pm 0.1$ \\
04850036 & 54011.96 & $3.8 \pm 0.3$ & $0.6 \pm 0.1$ \\
05370061 & 54168.41 & $4.1 \pm 0.2$ & $1.1 \pm 0.1$ \\
05370062 & 54168.45 & $4.6 \pm 0.5$ & $0.9 \pm 0.2$ \\
\hline
\end{tabular}

windows). Publicly available data mostly from GCDE observations during 2003-2005 have also been used.

The data have been reduced with the standard off-line analysis software (OSA) version 6.0. OSA is distributed and maintained by the INTEGRAL science data center $\left(\right.$ ISDC $\left.^{1}\right)$.

\subsection{Timing analysis}

The 20-40 keV lightcurve of XTE J1739-302 during GCDE in the period 2003-2005, and during KP 1 are shown in Figs. 1 and 2, respectively. During GCDE and KP1 observations there is clear flaring activity. In contrast, during the KP2 period no significant detection can be reported (but a 3- $\sigma$ upper limit on the source flux of 5 count s ${ }^{-1}$, that is $\sim 43 \mathrm{mCrab}$, can be given ${ }^{2}$ ). Times when INTEGRAL was observing in the source direction but when the source was not detected are indicated in Figs. 1 and 2 with zero flux (blue squares).

JEM-X detections in two energy bands (4-15 keV and 15$30 \mathrm{keV}$ ) are summarized in Table 1. Due to its smaller field of view (FOV) the total "on source" time is $\sim 100$ times smaller than that of INTEGRAL/ISGRI.

During the GCDE period, the coverage is sparse and the lack of homogeneity prevents the data from being useful for a statistically significant determination of a characteristic outburst frequency. There are three periods, however, during which the source was in the INTEGRAL/ISGRI FOV for a time interval long enough to ensure that there was no activity prior to or after the outburst activity. These periods are labelled A, B and C in Fig. 1. These activity periods have been reported and analyzed in Lutovinov et al. (2005), Sguera et al. (2005), Sguera et al. (2006), and Walter \& Zurita Heras (2007).

Coverage during the KP1 period is much more homogeneous, as can be seen in Fig. 2. We can define here 5 periods of continuous coverage, labelled E, F, G, H and I in Fig. 2. This light curve can be divided in 26 activity periods, following the definition used by Walter \& Zurita Heras (2007). In the majority of cases, these activity periods correspond to a strong peak and some associated flaring activity, with secondary peaks before and after the maximum flux of the peak. We call such an

\footnotetext{
1 http://isdc.unige.ch

2 The Crab flux in the $20-40 \mathrm{keV}$ energy band was obtained for 3 pointings of revolution 300 , namely pointings 39,40 and 41 , with OSA 6.0 software. A mean Crab flux of 117 count s$^{-1}$ was used to convert the XTE J1739-302 flux from count $\mathrm{s}^{-1}$ to Crab units.
} 


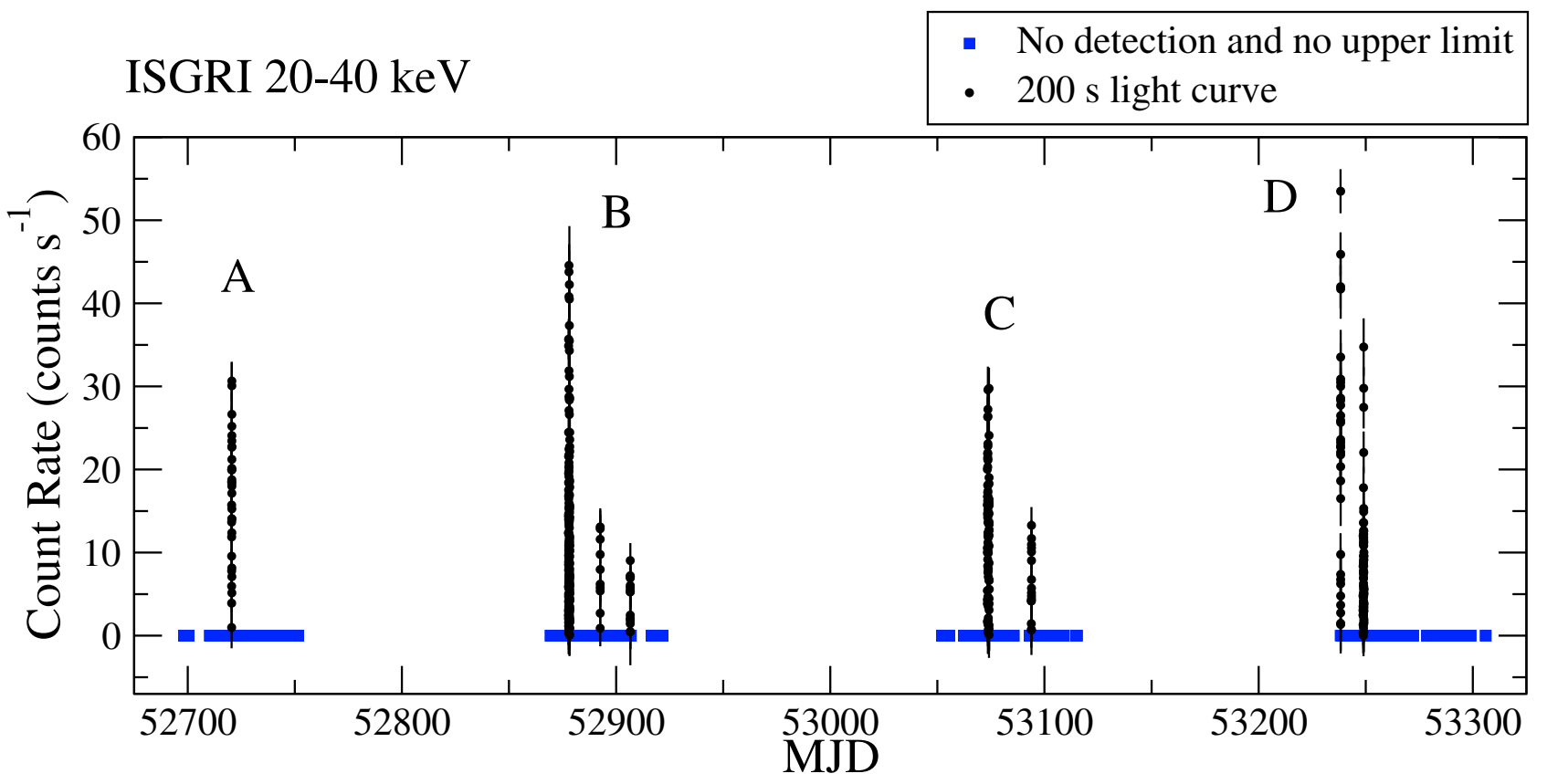

Fig. 1. Light curve of XTE J1739-302 data from the INTEGRAL Galactic Center Deep Exposure (GCDE). To reveal the number and structure of outbursts, a binning time of $200 \mathrm{~s}$ has been used.

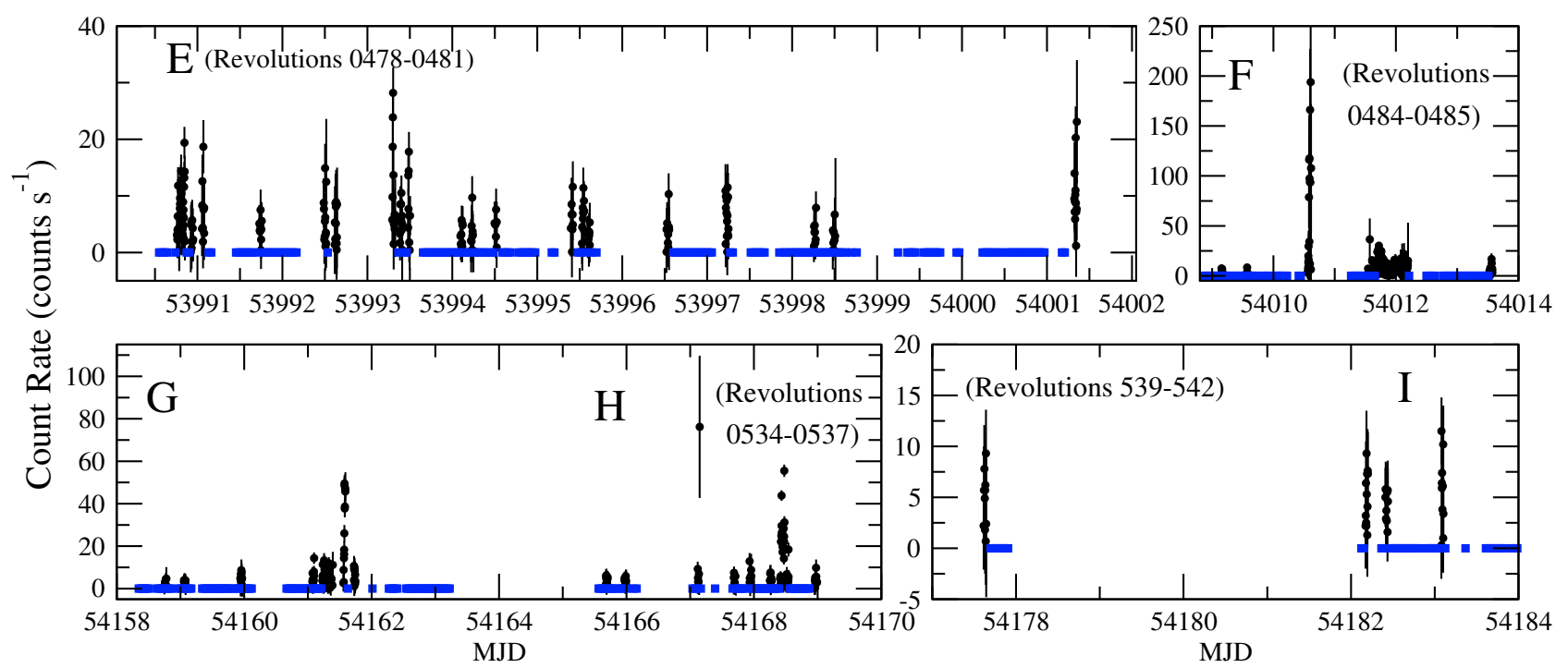

Fig. 2. Light curve of XTE J1739-302 data from INTEGRAL Key Programme 1 (KP1, September-November 2006 and February-March 2007). To reveal the number and structure of outbursts, a binning time of $200 \mathrm{~s}$ has been used.

activity period an outburst. In a few cases, like the 4 consecutive science windows from revolution 458 shown in Fig. 3, more than one strong peak fits within an activity period. The average duration of the activity periods is $\sim 6 \mathrm{~h}$, with a maximum duration of $16 \mathrm{~h}$ and a minimum duration of $0.6 \mathrm{~h}$. We can differentiate two types of outbursts: those with a mean count rate of $\sim 10$ count $\mathrm{s}^{-1}$, fainter and more numerous; and the brighter ones, less frequent and with an average flux of $\sim 60$ count $^{-1}$, but reaching $\sim 190$ count $\mathrm{s}^{-1}$. On average, both types of outburst last for a very similar time span, but the brightest ones tend to last for longer than $\sim 1 \mathrm{~h}$ and the faintest can be as short as $\sim 0.6 \mathrm{~h}$. This dataset can be used to search more consistently for a characteristic outburst frequency and we will do so in Sect. 3.1.

We have failed to detect any coherent modulation that could be associated with the pulse period, up to time scales of the order of $\sim 1000 \mathrm{~s}$. An apparent non-coherent modulation at about $4000 \mathrm{~s}$ is found in the KP1 data, see, e.g., the data for revolution 485 in Fig. 3. But this non-coherent modulation only reflects the characteristic time scale of the flaring activity during KP1, as described above. 


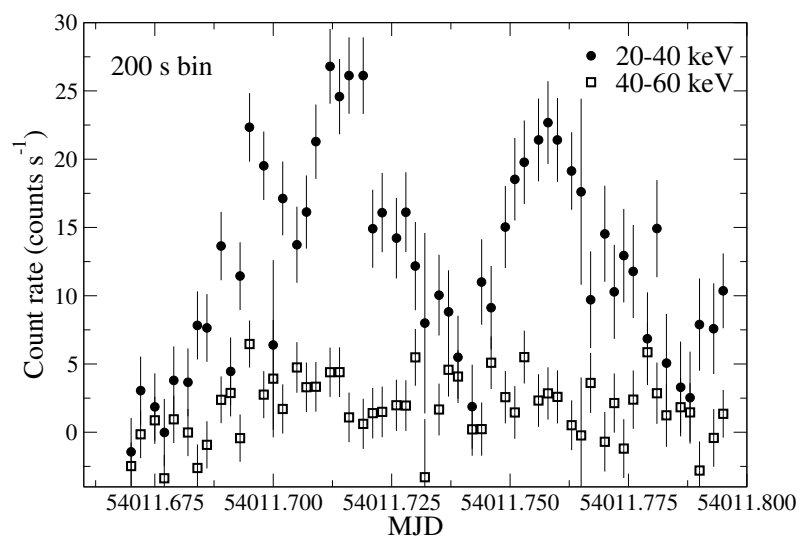

Fig. 3. Light curve of the four consecutive science windows (namely, $0027,0028,0029$, and 0030) of revolution 485, the longest set of consecutive observations in the KP1 period, with a total coverage of $8 \mathrm{ks}$. The time binning used for plotting purposes is $200 \mathrm{~s}$.

Table 2. Spectral parameters for the bremsstrahlung and powerlaw models fit of INTEGRAL/ISGRI data.

\begin{tabular}{lccccc}
\hline \hline Revolution & $\begin{array}{c}k T \\
(\mathrm{keV})\end{array}$ & $\chi_{\text {red }}^{2} /$ d.o.f & $\Gamma$ & $\chi_{\text {red }}^{2} /$ d.o.f & $\begin{array}{c}\text { Flux } \dagger \\
20-100 \mathrm{keV}\end{array}$ \\
\hline 0053 & $22_{-2}^{+3}$ & $0.8 / 20$ & $3.10_{-0.15}^{+0.16}$ & $1.0 / 20$ & 1.0 \\
0106 & $20_{-1}^{+2}$ & $1.6 / 20$ & $3.17_{-0.11}^{+0.11}$ & $1.9 / 20$ & 0.8 \\
$0171 \mathrm{a}$ & $20_{-2}^{+2}$ & $1.2 / 20$ & $3.14_{-0.14}^{+0.15}$ & $1.4 / 20$ & 1.0 \\
$0171 \mathrm{~b}$ & $20_{-4}^{+5}$ & $0.7 / 15$ & $3.2_{-0.3}^{+0.3}$ & $1.2 / 20$ & 0.7 \\
\hline 0478 & $22_{-5}^{+8}$ & $0.8 / 20$ & $3.1_{-0.4}^{+0.4}$ & $1.0 / 20$ & 0.5 \\
0485 & $18_{-2}^{+3}$ & $0.8 / 20$ & $3.28_{-0.19}^{+0.20}$ & $1.3 / 20$ & 0.7 \\
0535 & $21_{-3}^{+5}$ & $1.3 / 20$ & $3.04_{-0.23}^{+0.25}$ & $1.4 / 20$ & 1.0 \\
0537 & $25_{-3}^{+4}$ & $1.6 / 20$ & $2.89_{-0.16}^{+0.16}$ & $1.9 / 20$ & 1.1 \\
\hline
\end{tabular}

$\dagger \times 10^{-9} \mathrm{erg} \mathrm{cm}^{-2} \mathrm{~s}^{-1}$.

\subsection{Spectral analysis}

Only those science windows with the source within the fully coded field of view (FCFOV) of INTEGRAL/ISGRI and with a detection level above 7 for XTE J1739-302 were considered for the spectral extraction ${ }^{3}$. This leaves us with a total of 32 spectra extracted for the public data in the 2003-2005 period and a total of 12 spectra for the KP 1 period. These spectra correspond to the brightest part of the outbursts seen in Figs. 1 and 2. The spectra created per pointing have rather poor statistics and do not permit a detailed spectral analysis. We have therefore created average spectra per revolution, except for revolution 171 in which two spectra could be extracted with sufficient statistics to test for spectral variability.

A bremsstrahlung model (bremss in XSPEC notation) was used in order to search for spectral variability and to compare with previously published data (e.g., Smith et al. 1998). The spectral parameters are shown in Table 2. The fit results are, within the uncertainties, all compatible with a mean electron temperature of $21 \pm 2 \mathrm{keV}$ and no spectral variation, in good agreement with previously published values (see Smith et al. 2006; Lutovinov et al. 2005). The given uncertainties are calculated for a $90 \%$ confidence level.

\footnotetext{
3 The OSA software reports the ISGRI detections in a significance scale in which a source is considered as detected if its fitted with a detection level of 7 . This detection level of 7 is equivalent to the classical $3 \sigma$ level above the measured noise.
}

Even though simple bremsstrahlung models produced acceptable fits to the spectra, the residuals show the presence of possible absorption features at $\sim 30 \mathrm{keV}$ and $\sim 60 \mathrm{keV}$ in some of the spectra (see Fig. 4).

A powerlaw model was also used to fit the spectra, for comparison with other sources. The results are shown in Table 2. Apart from the possible absorption lines, which could not be seen in all spectra, no signs of spectral variability, within errors, could be found.

With the aim of investigating the spectral behaviour below $20 \mathrm{keV}$, we have made use of $R X T E / \mathrm{PCA}$ data (described in Smith et al. 1998) and INTEGRAL/JEM-X spectra from revolutions 53, 485 and 537. A spectrum from INTEGRAL/JEM-X data could also be extracted for revolution 171, but it was very noisy and not useful for spectral fitting. The RXTE/PCA spectrum together with the GCDE INTEGRAL/ISGRI average spectrum can be seen in Fig. 5. All joint spectra can be seen in Fig. 6. A powerlaw, modified by a photo-absorption at low energies plus a cut-off at high energies, was used as the basic model and the photo-absorption column was fixed to $4.2 \times 10^{22} \mathrm{~cm}^{-2}$ (Smith et al. 1998) in all cases and the cross-calibration factor was set to unity for RXTE/PCA and for INTEGRAL/JEM-X spectra.

A summary of the fit of these joint PCA/JEM-X-ISGRI spectra is shown in Table 3. Uncertainties and upper limits are given at a $90 \%$ confidence level.

Regardless of the continuum models used, for the datasets of revolutions 53,106, and 478, we see residual features reminiscent of broad absorption structures at $\sim 30 \mathrm{keV}$ for the former, $\sim 65 \mathrm{keV}$ for revolution 106 and $\sim 30 \mathrm{keV}$ and $\sim 60 \mathrm{keV}$ for the latter (see Fig. 4). It is tempting to associate them with a cyclotron resonant scattering feature (CRSF) and its first harmonic, but adding such features to the spectral model does not lead to a significant improvement of the fit. A deeper exposure of the source would be required in order to obtain sufficient statistics for an analysis of these possible spectral features.

\section{Discussion}

\subsection{Outburst frequency}

Walter \& Zurita Heras (2007) attempt to define a typical flare duration in SFXTs, in order to quantify the frequency of outbursts. They identify a typical flare duration of $3 \mathrm{~h}$, though flares are sometimes seen to be part of longer flaring episodes. Walter \& Zurita Heras (2007) define a flaring episode as separated by at least $25 \mathrm{ks}$ of non-detection from another episode. During the time span 2003-2005, they count a total of 13 of these flaring episodes from XTE J1739-302 detected during a total observed elapsed time of $126.4 \mathrm{~d}$. Of these, only one can be interpreted as a longer $(\sim 14 \mathrm{~h})$ flare, but it is also possible to interpret it as a superposition of three shorter flares. The rest of the flares last between $\sim 2$ and $\sim 8 \mathrm{~h}$, with a typical duration of $4.2 \mathrm{~h}$, similar to other SFXTs. In any case, due to the non-continuity of the observations it is difficult to set a characteristic outburst frequency for this period.

Data during the KP1 observations offer a much more homogeneous coverage. Table 4 summarizes the outburst counting, showing the duration of the quasi-continuous observing period, the number of outbursts, as seen in Fig. 2, and the derived outburst frequency for each period. This is obtained by simply dividing the number of outbursts seen by the time duration of the observing period (considering only the "on-source" time).

The results are surprising, because the outburst frequency derived is much higher than previously expected (2-4 outbursts $\mathrm{d}^{-1}$ 

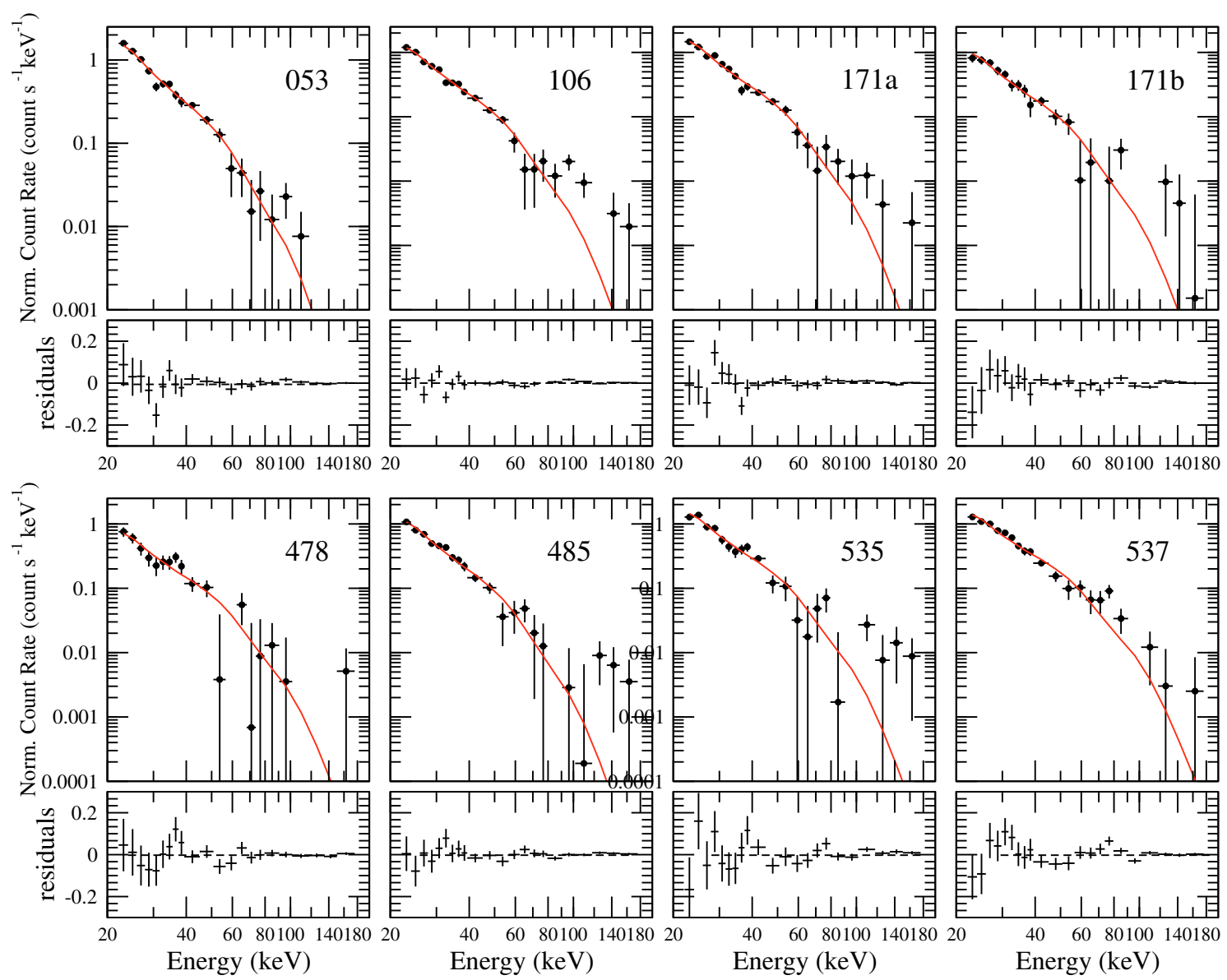

Fig. 4. Bremsstrahlung fit to the average INTEGRAL/ISGRI spectra of revolutions shown in Table 2. The corresponding revolution number is indicated in the top right corner of each plot. The fit shown is that of the bremsstrahlung model. Residuals are in units of count $\mathrm{s}^{-1} \mathrm{keV}^{-1}$.

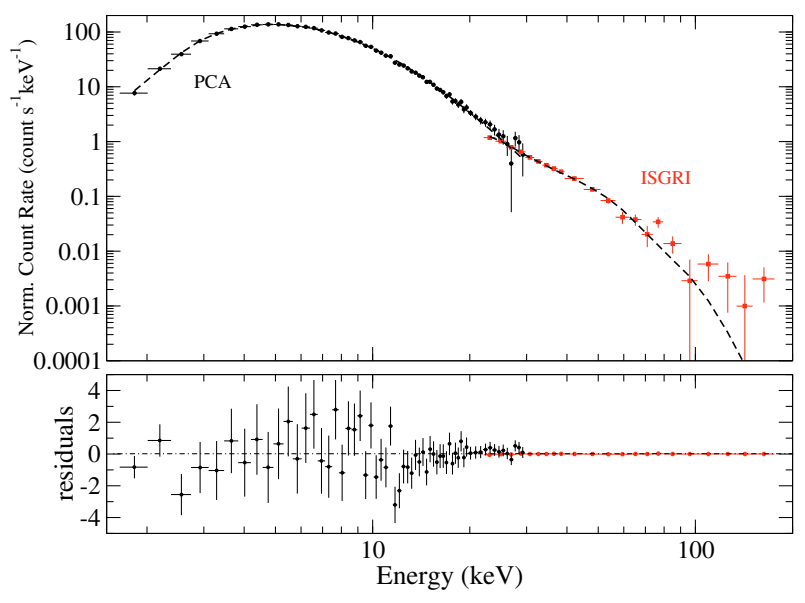

Fig. 5. Fit to the joint $R X T E / P C A$ plus INTEGRAL/ISGRI average spectrum. Residuals are in units of count $\mathrm{s}^{-1} \mathrm{keV}^{-1}$.

as opposed to 0.14 outbursts $\mathrm{d}^{-1}$ given as average of INTEGRAL detections of SFXTs by Walter \& Zurita Heras 2007) and does not seem to vary much between observations. This higher frequency is also seen in the active periods during the GCDE observations, such as the MJD 52 877-78 and 53 073-74 intervals in Fig. 1.

The average frequency for the KP1 observations is 3.5 outburst $\mathrm{d}^{-1}$. However, no outbursts were seen during the KP2 observations. Examination of Fig. 1 shows that long time intervals are characterized by low X-ray activity. We know that the absence of activity is not complete, as at least one outburst from XTE J1739-302 was observed by the RXTE/PCA during its Galactic Bulge Scan observations on 54347.5 MJD. This outburst took place during the long gap in the I bin of KP2 observations ${ }^{4}$.

Taking the average outburst frequency for KP1 at face value $^{5}$, we can compute the orbital period of a compact object necessary to match the observed frequency, as a function of the radial distance. This can be accomplished by taking into account that

$v_{\text {outb }}=\frac{N(r)_{\mathrm{c}}^{\text {ring }}}{P_{\text {orb }}}$

where $N_{\mathrm{c}}^{\text {ring }}$ is the number of clumps inside a ring at a distance $r$ from the donor star. This quantity represents the number of clumps that a NS orbiting in a circular orbit of radius $r$ will statistically be able to "see" (and accrete, see Negueruela et al. 2008). The $P_{\text {orb }}$ vs. $v_{\text {outb }}$ relationship is depicted in the left panel of Fig. 7 by the solid line.

\footnotetext{
${ }^{4}$ Craig Markwardt, private communication.

5 If KP2 was carried out during an unusually inactive period of this source while KP1 occurred during an unusually high activity period, it would make sense to consider an average outburst frequency of $v_{\text {outb }} \approx$ $1.5 \mathrm{~d}^{-1}$. Then, maintaining XTE J1739-302 outside the smooth wind zone of Fig. 7 (left panel) would require $L_{0} \approx 0.25$. The values of $r$ and $P_{\text {orb }}$ do not change too much and the terms of the discussion are nearly the same.
} 


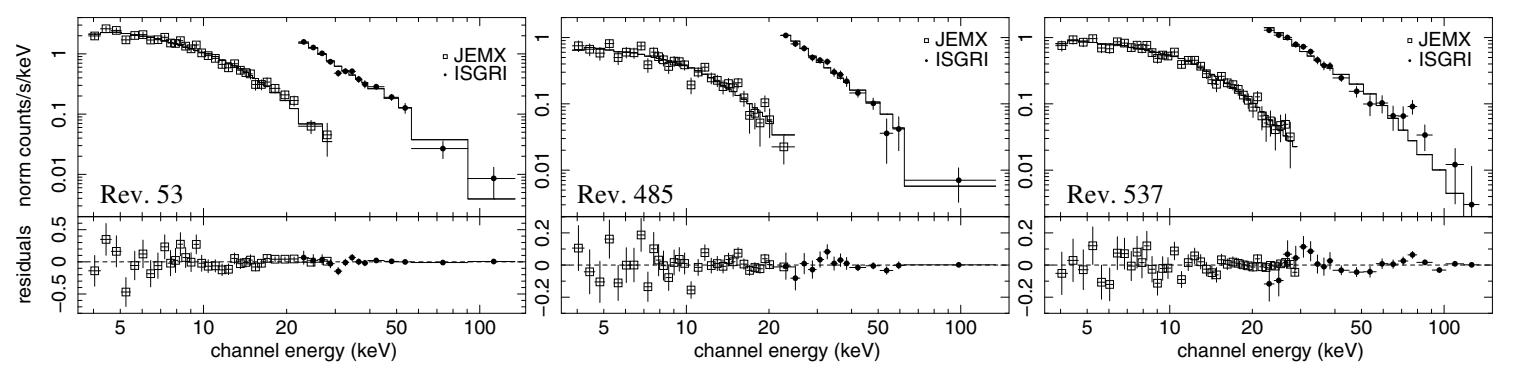

Fig. 6. Fit to the joint INTEGRAL/JEM-X plus INTEGRAL/ISGRI spectra from revolutions 53, 485, and 537. Residuals are in units of count $\mathrm{s}^{-1} \mathrm{keV}^{-1}$.

Table 3. Summary of best fit parameters obtained when joining RXTE/PCA data together with the GCDE INTEGRAL/ISGRI average spectrum, and INTEGRAL/ISGRI and INTEGRAL/JEM-X from revolutions 53, 485, and 537.

\begin{tabular}{ccccccc}
\hline \hline $\begin{array}{c}\text { Joint } \\
\text { Spectra }\end{array}$ & $\begin{array}{c}N_{\mathrm{H}} \\
\times 10^{22} \mathrm{~cm}^{-1}\end{array}$ & $\begin{array}{c}\text { Cross-Calibration } \\
\text { Constant }\end{array}$ & $\Gamma$ & $\begin{array}{c}E_{\text {cut }} \\
(\mathrm{keV})\end{array}$ & $\begin{array}{c}E_{\text {fold }} \\
(\mathrm{keV})\end{array}$ & $\chi_{\text {red }}^{2}$ /d.o.f. \\
\hline PCA+ISGRI & 4.2 & $0.59 \pm 0.03$ & $1.24 \pm 0.04$ & $6.2 \pm 0.5$ & $18 \pm 1$ & $1.0 / 79$ \\
\hline Rev. 053, JEM-X+ISGRI & 4.2 & $0.80 \pm 0.10$ & $1.80 \pm 0.11$ & $<11$ & $29_{-5}^{+6}$ & $1.00 / 56$ \\
Rev. 485, JEM-X+ISGRI & 4.2 & $1.8 \pm 0.3$ & $1.7 \pm 0.2$ & $<11$ & $22_{-4}^{+6}$ & $1.03 / 42$ \\
Rev. 537, JEM-X+ISGRI & 4.2 & $1.3 \pm 0.2$ & $1.56 \pm 0.11$ & $<11$ & $28_{-5}^{+6}$ & $1.10 / 56$ \\
\hline
\end{tabular}

Table 4. Frequency of outbursts for the activity periods defined during KP1 observations.

\begin{tabular}{lcccc}
\hline \hline Period & Interval & $\begin{array}{c}\text { On source } \\
\text { time } \\
(\mathrm{d})\end{array}$ & $\begin{array}{c}\text { Num. of } \\
\text { outburst }\end{array}$ & $\begin{array}{c}\text { Outburst } \\
\text { Frequency } \\
\left(\mathrm{d}^{-1}\right)\end{array}$ \\
\hline $\mathrm{E}$ & $53990.50-54001.50$ & 5.5 & 21 & 3.8 \\
$\mathrm{~F}$ & $54008.75-54014.00$ & 3.1 & 13 & 4.2 \\
$\mathrm{G}$ & $54158.00-54163.25$ & 3.0 & 8 & 2.7 \\
$\mathrm{H}$ & $54165.50-54169.00$ & 1.8 & 8 & 4.4 \\
$\mathrm{I}$ & $54182.00-54184.00$ & 1.3 & 3 & 2.3 \\
\hline
\end{tabular}

On the other hand, we can calculate the orbital period of a binary system by simply applying Kepler's third law, and express it as a function of binary separation. This has been depicted as a dashed line in Fig. 7 for a NS of canonical mass $1.4 M_{\odot}$ orbiting a O8 I star.

For the high frequency observed, the clumping parameter $L_{0}$ (Oskinova et al. 2007; Negueruela et al. 2008) must have a value of $\sim 0.2$, close to the lower bound of the range suggested by Oskinova et al. (2007) (0.2-0.5) based on independent optical and ultraviolet observations. For higher values of $L_{0}$ the system would lie in the smooth wind regime.

In Negueruela et al. (2008) we stated that a value of $L_{0} \approx$ 0.35 was adequate outside the smooth regime zone to reproduce the observed properties of some fast transient systems. However, we argued, its value must be lower $\left(L_{0} \approx 0.1\right)$ inside the asymptotic zone in order to produce the persistent emission seen in the classical systems. One possibility to circumvent this discrepancy is to consider a radial dependence of this parameter, increasing outwards. If this is the case, it is entirely natural that, in the (narrow) transition zone - from the smooth wind regime to the fully developed clumps - where XTE J1739-302 orbits, presents an intermediate value.

Under this interpretation, the system therefore should have an orbital separation of $r \lesssim 3 R_{*}$ and a $P_{\text {orb }} \approx 8 \mathrm{~d}$. The proximity of the NS to the smooth regime zone is consistent with the high activity displayed by the source.
Inspection of Fig. 2 shows that the vast majority of outbursts have an average maximum flux of $\sim 10-12$ counts $\mathrm{s}^{-1}$. Superimposed on this "flaring activity" (faintest outbursts) there are outbursts with a maximum flux several times higher than this flaring level. For example, in bin E, we have two outbursts that surpass the 20 counts $\mathrm{s}^{-1}$ barrier. In bin $\mathrm{F}$, we have one with an outstanding $\sim 200$ counts $\mathrm{s}^{-1}$. In bins $\mathrm{G}$ and $\mathrm{H}$, we have two outbursts reaching $\sim 50-60$ counts $\mathrm{s}^{-1}$. The flaring in bin $\mathrm{I}$ is again normal.

The distance to this source is $d=2.3 \mathrm{kpc}$ (Negueruela et al. 2006b). Therefore these fluxes translate into the following luminosities: the flaring activity is at a level of $\sim 5 \times 10^{35} \mathrm{erg} \mathrm{s}^{-1}$. The two outbursts in bin E reach luminosities of $\sim 10^{36} \mathrm{erg} \mathrm{s}^{-1}$ and those in bins $\mathrm{G}$ and $\mathrm{H} \sim 2.5 \times 10^{36} \mathrm{erg} \mathrm{s}^{-1}$. Finally, the strong outburst in bin $\mathrm{F}$ reaches $\sim 10^{37} \mathrm{erg} \mathrm{s}^{-1}$. In other words, the bright outbursts are between 2 and 20 times more powerful than the faint ones. Otherwise, they seem to be identical. The bright outbursts seem to be regularly separated by an interval of $\sim 8 \mathrm{~d}$ (MJDs 53 993.5, 54 001.4, 54 0010.5, 54 160.7, 54 168.5), which coincides with the orbital period deduced from our model $^{6}$. In the following lines, and with the aims to make the text clearer, with outburst we will mean only the brightest ones, and will refer to the rest as "flares" or "flaring activity" periods.

To investigate whether these luminosities can fit into our scenario, we have plotted the accretion luminosity of simple BondiHoyle wind accretion, following the formalism described in Reig et al. (2003). This is plotted in the right panel of Fig. 7. We have chosen an efficiency factor of $\eta=0.25$ appropriate for accretion on to a NS. Furthermore, we have applied a multiplicative factor adjusted to match the range of observed luminosities in classical supergiants (namely, $\left[10^{36}-10^{37}\right] \mathrm{erg} \mathrm{s}^{-1}$ ), inside the smooth wind zone. At $r=2 R_{*}$ we have $L_{\mathrm{X}}=2 \times 10^{36} \mathrm{erg} \mathrm{s}^{-1}$, as observed in the O8-9 Ia star 4U 1907+09 (Cox et al. 2005). We also show the predicted luminosities increased or decreased by a factor of 10 . Now, assuming that the putative NS is at $r \lesssim 3 R_{*}$, we can see that the luminosities predicted by this model agree very well with those observed in the flaring activity. In other words,

\footnotetext{
${ }^{6}$ And also with the outburst frequency found by Walter \& Zurita Heras (2007). These authors were detecting only the strong outbursts.
} 

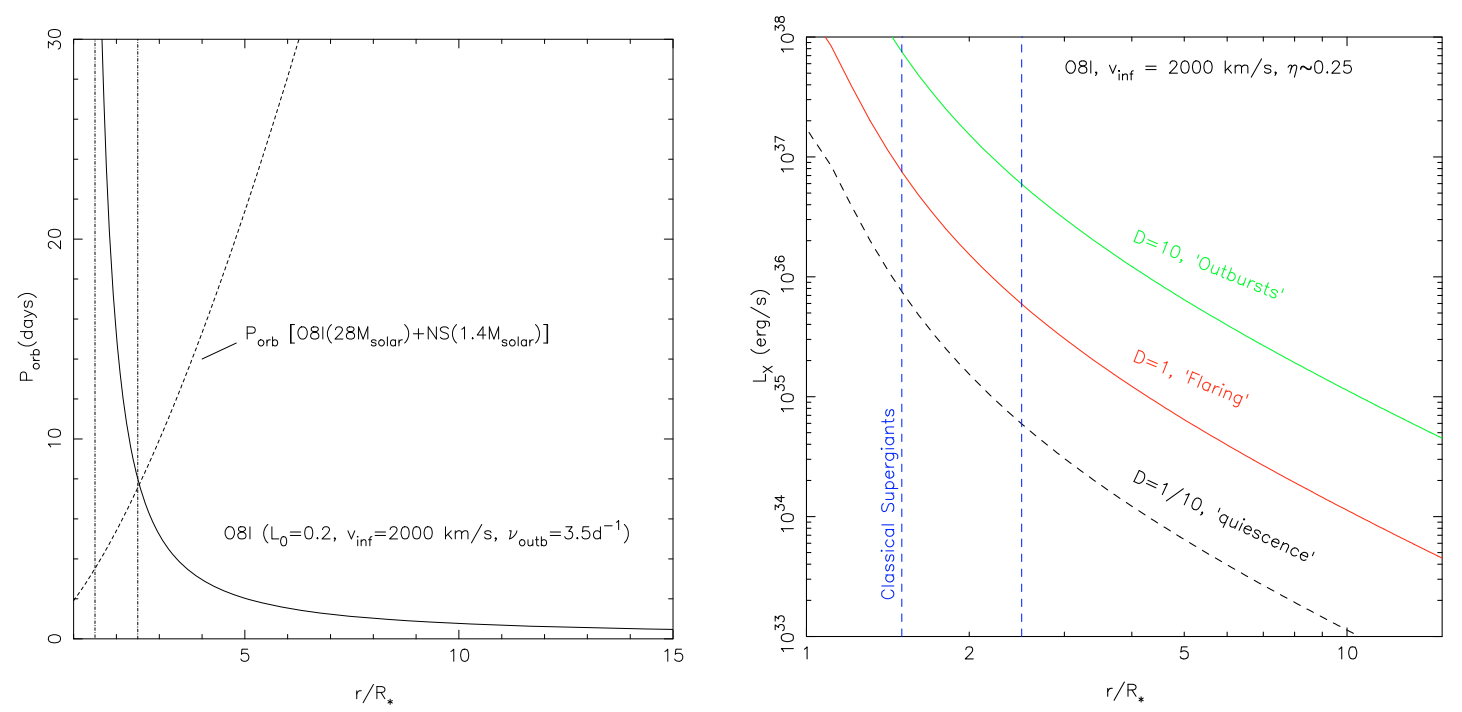

Fig. 7. Left panel: orbital periods corresponding to a certain outburst frequency ( 3.5 per day, in this case) versus the radial distance (solid line). The dashed line represent the Kepler law for a NS orbiting an O8I star. Vertical lines mark the smooth wind regime region, where the classical supergiants reside. For the observed frequency, the NS must lie close to the smooth region upper limit, at $r \lesssim 3 R_{*}$. Right panel: accretion luminosity versus radial distance. At the distance to the system, the luminosity is $\sim 5 \times 10^{35} \mathrm{erg} \mathrm{s}^{-1}$, as observed in the flares. Relative changes in the density by a factor of 10 (plus or minus) can led to outburst or "quiescence".

the majority of clumps have the same density and velocity predicted by the smooth wind approximation at this distance. In order to produce the outbursts we only need to invoke increments in the density of the clumps by the corresponding factors 2 to 20. On the other hand, a decrease in density of about the same factor would bring the source under the level of detectability. Therefore, density contrasts of $\sim 10^{1}-10^{2}$ would explain all but the strongest outburst. Such contrasts are lower than expected in most formulations of the porous wind model (e.g., Runacres \& Owocki 2002; Owocki \& Cohen 2006).

During KP2, no outbursts or flaring activities were detected. We are able to set a 3- $\sigma$ upper limit on the flux of $43 \mathrm{mCrab}$. This corresponds to a luminosity of $\sim 2.5 \times 10^{35} \mathrm{erg} \mathrm{s}^{-1}$. Since this upper limit in KP2 is close to the flaring level in KP1, the lack of flaring detection could not mean, necessarily, a real lack of activity (in fact, the upper limit found for the luminosity is well above the typical quiescence luminosity reported by Sidoli et al. 2008). A drop in the density of the wind by only a factor of 2.5 could bring the flares already under the level of detectability. As mentioned above, RXTE observed a flare during the long gap in the KP2 observations.

The lack of outbursts is more complicated to explain. While the lack of any detection during KP2 would still be consistent with the presence of a low luminosity flaring (induced by a decrease in wind density, for example), the lack of outbursts is real.

We have seen before that these outbursts seem to be separated by $\sim 8 \mathrm{~d}$, very similar to the predicted orbital period. In principle, the most straightforward explanation is that these are produced at or close to the periastron passage on an eccentric orbit. In order to reproduce the ratio of luminosities betwen flares and the outbursts (a factor of 2 to 5 , not considering the large outburst of bin F) would require a degree of eccentricity of 0.1 . However, there is no special reason why we should observe no outbursts during KP2.

\subsection{Lack of pulsations}

In principle, the possibility that the compact object in XTE J1739-302 is a black hole cannot be completely discarded.
However, due to the photon index found in the spectral fit and the marginal evidence of a possible CRSF we will consider in the following discussion that the compact component in XTE J1739302 is a NS. If this is the case, the absence of pulsations can be explained by geometrical effects. This possibility has also been considered in order to explain the lack of pulsations in 4U 170037 (White et al. 1983), 4U 1700+24 (Masetti et al. 2002), and 4U 2206+54 (Blay et al. 2005). Two scenarios can be invoked: a) in the first one, the magnetic axis and the spin axis of the NS are almost aligned, in this configuration only one pole of the NS is continuously visible; b) the second scenario would imply that the orbit of the binary system has a very small inclination angle.

Furthermore, the possibility of very slow pulsations cannot be excluded. Although our data set is not suitable for a proper timing analysis, because of the many gaps between the observations, we have not found evidence of pulsations on time scales up to $1000 \mathrm{~s}$. Smith et al. (1998) discarded the presence of pulsations below 300 s. 2S 0114+650, an X-ray pulsar accreting matter from the wind of a supergiant B1 companion (Reig et al. 1996), shows pulsations with a spin period of $2.8 \mathrm{~h}$ (Finley et al. 1994). Another example of a neutron star showing very slow pulsations is the case of that in the low mass X-ray binary system $4 \mathrm{U} 1954+319$, which pulsates with a period of $5 \mathrm{~h}$ (Mattana et al. 2006; Corbet et al. 2008). The inhomogeneity of our data at this time scale hinders the detection of a modulation of this order of magnitude.

We have considered that the X-ray variability directly traces the supergiant wind behaviour, however the accretion process could be more complex and, then, our conclusions could not be derived in such a straightforward manner. Grebenev \& Sunyaev (2007) suggest that the flaring behaviour of fast X-ray transients can be due to the onset and offset of the propeller effect in those systems with a NS companion. This would happen in systems in which the NS possesses a spin period close to a certain limiting value $P_{\text {prop. }}$. However if the absoption features suggested by our data at the energies of $\sim 30 \mathrm{keV}$ and $\sim 60 \mathrm{keV}$ correspond to a real CRSF and its first harmonic, that would imply a magnetic field strength of the order of $\sim 3 \times 10^{12} \mathrm{G}$ (according to the formula $\left[B / 10^{12} \mathrm{G}\right]=[E / \mathrm{keV}](1+z) / 11.6$, which relates the energy of 
the CRSF, $E$, to the magnetic field strength, $B$, and where $z$ represents the gravitational red-shift at which we see the absorbing region). This value is well within the range $1.3-4.8 \times 10^{12} \mathrm{G}$ for the expected magnetic field strength of a NS obtained by Coburn et al. (2002). The NS in XTE J1739-302 does not seem to rotate at a particularly fast rate (periods below $1000 \mathrm{~s}$ are discarded) and possibly shows a typical value for its magnetic field strength. Thus, we do not find reasons why the accretion process in this system should be different from the majority of accreting systems and why the propeller effect could be present. Furthermore, if we take the typical values of $m_{\mathrm{NS}}=1.4 M_{\odot}, R_{\mathrm{NS}}=10 \mathrm{~km}$, $v_{\mathrm{NS}}=10^{3} \mathrm{~km} \mathrm{~s}^{-1}$ for the mass, radius and relative velocity to the stellar wind of the neutron star, and we take into account the estimated possible magnetic field of $\sim 3 \times 10^{12} \mathrm{G}$ and the possible $8 \mathrm{~d}$ orbital period, we obtain, according to the formalism of Grebenev \& Sunyaev (2007), that only for spin periods faster than $\sim 10 \mathrm{~s}$ would the propeller effect take place, for mass loss rates from the donor star of the order of $\sim 10^{-5} M_{\odot} \mathrm{yr}^{-1}$. The possibility of reaching this limiting $P_{\text {prop }}$ is ruled out by the fact that pulse periods shorter than $\sim 1000 \mathrm{~s}$ are discarded. The derivation of this limiting value is done in the framework of the smooth wind approximation and, therefore, may have to be reformulated in order to account for the peculiarities of the clumpy wind formalism.

Titarchuk et al. (2002) proposed the smearing out of pulsations due to electron scattering in an optically thick environment. This model was already considered as a possible explanation for the lack of pulsations in the HMXRB 4U 2206+54 by Torrejón et al. (2004). Due to the high absorption column found in XTE 1739-302 (Smith et al. 1998), the possibility of this mechanismoperating in this system should not be discarded.

\section{Conclusions}

XTE J1739-302 has been observed with the INTEGRAL observatory over a long time span. In the period 2003-2005, as part of the INTEGRAL core program observations of the Galactic Center, the source showed moderate activity, with an average outburst frequency below 1 outburst per day. During the deep exposures of the Galactic Center taken within the frame of our first Key Programme observations, in the 2006-2007 period, XTE J1739-302 showed a higher level of activity, with a mean outburst frequency of $\sim 3$ outbursts per day. Surprisingly, during the last observations in 2007 (within the second run of our Key Programme) the source showed an unusually low activity state, and no outburst was detected with a flux above $43 \mathrm{mCrab}$. The behaviour during the first two periods can be explained well within the framework of the clumpy wind models. These models will not only explain the observed properties of the source but will also predict an orbital periodicity around $\sim 8 \mathrm{~d}$ and suggests the presence of a NS as the compact companion. To explain the low activity observed during the KP2 period, geometrical considerations related to the eccentricity of the orbit, or a drop in the mass loss from the supergiant companion, need to be invoked in order to maintain consistency with the proposed model. A continuous monitoring of the system will allow disentangling of which of the three observed behaviours (non dectability, moderate activity, and high activity) represents the normal state of the source, will lead to a careful determination of the model parameters, and will permit us to constrain the geometrical parameters of the system.

An independent constraint or measure of the orbital period of XTE J1739-302 would help to support or discard the clumpy wind model as the explanation of the behaviour of
XTE J1739-302. This model would, then, be the first consistent attempt to explain the observational properties of the SFXT and the classical wind-fed supergiant systems all together. The long spans of high activity seem incompatible with the model proposed by Sidoli et al. (2007), where outbursts happen once or twice each orbital cycle. Moreover, the observations presented here represent strong evidence against a coherent periodicity in the recurrence of the outbursts, which is a requirement of the model. This model was specifically designed for IGR J11215-5952, a system presenting periodic outbursts, and its applicability to SFXTs is just a hypothesis, even if it turns to be appropriate for this particular source.

Acknowledgements. We are grateful to Craig Markwardt for sharing RXTE data durin the INTEGRAL KP2 period.

S.M.N. is a researcher of the Programme Juan de la Cierva, funded by the Spanish Ministerio de Educación y Ciencia (MEC) and the University of Alicante, with partial support from the Generalitat Valenciana and the European Regional Development Fund (ERDF/FEDER). This research is partially supported by the MEC under grants AYA2005-00095 and CSD2006-70.

J.M.T. aknowledges the support by the Spanish Ministerio de Educación y Ciencia (M.E.C.) under grant PR2007-0176.

P. R. acknowledges the support of the European Union Marie Curie grant MTKD-CT-2006-039965.

This research has made use of data obtained through the INTEGRAL Science Data Center (ISDC), Versoix, Switzerland.

\section{References}

Blay, P., Ribó, M., Negueruela I., et al. 2005, A\&A, 438, 963 Chaty, S., \& Filliatre, P. 2005, ChJAS, 5, 104

Coburn, W., Heindl, W. A., Rotschild, R. E., et al. 2002, ApJ, 580, 394 Corbet, R. H. D., Sokoloski, J. L., Mukai, K., et al. 2008, ApJ, 675, 1424 Cox, N. L. J. , Kaper, L., \& Mokiem, M. R. 2005, A\&A 436, 661 Finley, J. P., Taylor, M., \& Belloni, T. 1994, ApJ, 429, 356 Forot, M., Laurent, P., Lebrun, F., et al. 2007, ApJ, 668, 1259 Fritz, S., Kreykenbohm, I., Wilms, J., et al. 2006, A\&A, 458, 885 Grebenev, S. A., \& Sunyaev, R. A. 2007, Ast. Lett., 33, 149 Krivonos, R., Produit, N., Kreykenbohm, I. et al. 2003, ATel, 211 Labanti, C., Di Cocco, G., Ferro, G., et al. 2003, A\&A, 411, 149 Laurent, P., Paul, J., Denis, M., et al. 1995, A\&A, 300, 399 Leahy, D. A. 2002, A\&A, 391, 219

Lebrun, F., Leray, J. P., Lavocat, P., et al. 2003, A\&A, 411, 141

Lei, F., Green, A. R., Bird, A. J., et al. 1997, Proc. 2nd INTEGRAL Workshop, ESA SP-382, 643

Lund, N., Budtz-Jørgensen, C., Westergaard, N. J., et al. 2003, A\&A., 411, L231 Lutovinov, A., Revnivtsev, M., Molkov, S., et al. 2005, A\&A, 430, 997

Masetti, N., Dal Fiume, D., Cusumano, G., et al. 2002, A\&A, 382, 104

Mattana, F., Götz, D., Falanga, M., et al. 2006, A\&A, 460, 1

Negueruela, I., Smith, D. M., Reig, P., et al. 2006a, ESA SP-604, 165

Negueruela, I., Smith, D. M., Harrison, T.E., \& Torrejón, J. M. 2006b, ApJ, 638, 982

Negueruela, I., Torrejón, J. M., Reig, P, et al. 2008,

[arXiv: 0801.3863]

Oskinova, L.M., Hamann, W.-R., \& Feldmeier, A. 2007, A\&A, 476, 1331

Owocki, S. P., \& Cohen, D. H. 2006, ApJ, 648, 565

Ribó, M., Negueruela, I., Blay, P., et al. 2006, A\&A, 449, 687

Reig, P., Chakrabarty, D., Coe, M. J., et al. 1996, A\&A, 311, 879

Reig, P., Ribó, M., Paredes J. M., et al. 2003, A\&A, 405, 285

Runacres, M. C., \& Owocki, S. P. 2002, A\&A, 381, 1015

Sguera, V., Barlow, E. J., Bird, A. J., et al. 2005, A\&A, 444, 221

Sguera, V., Bazzano, A., Bird, A. J., et al. 2006, ApJ, 646, 452

Sidoli, L., Romano, P., Mereghetti, S., et al. 2007, A\&A, 476, 1307

Sidoli, L., Romano, P., Mangano, V., et al. 2008, [arXiv: 0805. 1808]

Smith, D. M., Main, D., Marshall, F., et al. 1998, ApJ, 501, L181

Smith, D. M., Heindl, W. A., Markwardt, C. A., et al. 2006, ApJ, 638, 974

Titarchuk, L., Cui, W., \& Wood, K. 2002, ApJ, 576, 49

Torrejón, J. M., Kreykenbohm, I., Orr, A., et al. 2004, A\&A, 423, 301

Ubertini, P., Lebrun, F., Di Cocco, G., et al. 2003, A\&A, 411, L131

Vedrenne, G., Roques, J.-P., SchÃúnfelder, V., et al. 2003, A\&A, 411, L63

Walter, R., \& Zurita Heras, J. A. 2007, A\&A, 476, 335

White, N. E., Swank, J. H., \& Holt, S. S. 1983, ApJ, 270, 711

Winkler, C., Courvoisier, T. J.-L., Di Cocco, G., et al. 2003, A\&A, 411, L1 